International Journal of Pure and Applied Mathematics

Volume 83 No. 1 2013, 121-135

ISSN: 1311-8080 (printed version); ISSN: 1314-3395 (on-line version)

url: http://www.ijpam.eu

doi: http://dx.doi.org/10.12732/ijpam.v83i1.11

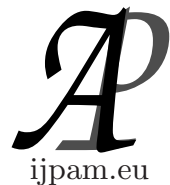

\title{
MINIMAL IDEALS OF ABEL-GRASSMANN GROUPOIDS
}

\author{
Madad Khan ${ }^{1}$, K.P. Shum ${ }^{2}$, M. Faisal Iqbal ${ }^{3}$ \\ ${ }^{1,3}$ Department of Mathematics \\ COMSATS Institute of Information Technology \\ Abbottabad, PAKISTAN \\ ${ }^{2}$ Institute of Mathematics \\ Yunan University \\ Kunming, 650091, P.R. CHINA
}

\begin{abstract}
The properties of Abel-Grassmann groupoids have been attracted the attention of many authors. The aim of this paper is to study the properties of the minimal left ideals of an Abel-Grassmann groupoid ( in brevity, an $A G$ groupoid ) with left identity. It is proved that if $L$ is a minimal left ideal of an $A G$-groupoid $S$ with left identity then $L c$ is a minimal left ideal of $S$ for all $c \in S$. We also show that the kernel $K$ of an $A G$-groupoid $S$ (the intersection of all two sided ideals of $S$ if exists)is simple and the class sum $\Sigma$ of all minimal left ideals of $S$ containing at least one minimal left ideal of $S$ is precisly the kernel $K$ of $S$. Finally, we show that if $S$ is an $A G$-groupoid with left identity then $S a^{2} S=S a^{2}$ for all $a \in S$. Finally, if $S$ is an $A G$-groupoid with left identity and does not contain any non-trivial nilpotent ideals, then every minimal ideal of $S$ is simple.A number of classical results of L. M. Gluskin and O. Steinfeld given in 1978 [3] concerning the minimal one sided ideals of semigroups and rings are consequently extended to and strengthened in $A G$-groupoids.
\end{abstract}

AMS Subject Classification: 20M10, 20N99

Key Words: AG-groupoids, medial law, minimal ideals, 0-minimal ideals, nilpotent ideals

Received: November 29, 2012

(C) 2013 Academic Publications, Ltd. url: www.acadpubl.eu

${ }^{\S}$ Correspondence author 


\section{Introduction}

Throughout this paper, unless otherwise state,we denote an $A G$-groupoid by $S$. In the literature, a left almost semigroup (in brevity, a $L A$-semigroup) is said to be an Abel-Gassmann groupoid (i.e. an $A G$-groupoid). By an $A G$-groupoid $S$, we mean a groupoid satisfying the following left invertive law

$$
(a b) c=(c b) a, \quad \text { for all } a, b, c \in S .
$$

The following medial law is always satisfied in $S$ :

$$
(a b)(c d)=(a c)(b d), \quad \text { for all } a, b, c, d \in S .
$$

It is known that the left identity of a groupoid $S$ if exists is unique [8].

In an $A G$-groupoid $S$ with left identity, the following paramedial law holds.

$$
(a b)(c d)=(d b)(c a) \text { holds, for all } a, b, c, d \in S .
$$

We recall that an $A G$-groupoid is a non-associative algebraic structure which is mid way between a groupoid and a commutative semigroup. A groupoid satisfying the above equations (2) and (3) is called a medial and paramedial groupoid respectively, see [5] and [6].It is interesting to note that an $A G$ groupoid $S$ with a left identity is a medial and paramedial groupoid. However, an $A G$-groupoid with a right identity will be a commutative semigroup with an identity [7]. We now call $S$ a locally associative $A G$-groupoid if for all $a \in S$, the equality $(a a) a=a(a a)$ holds. The general properties of $A G$-grouoids have been studied by P. V. Protic and N. Stevanovic in [20] and [21]. We notice that the $A G$-groupoids and the left regular partial ordered $A G$-groupoids have also been investigated and discussed in [16],[19] and [17]. In an $A G$-groupoid $S$, the following equality holds.

$$
(a(b c)=b(a c) \quad \text { for all } a, b, c \in S
$$

We now call an $A G$ - groupoid an $A G^{* *}$ - groupoid.

The above groupoid was first studied by Q. Iqbal and Q. Mushtaq [15] that the equality

$$
(a b)^{n}=a^{n} b^{n}
$$

holds for all $a, b \in S$ in a locally associative $A G$-groupoid $S$. Also,it has been proved in [16] that if $S$ is a locally associative $A G^{* *}-$ groupoid, then $a^{n} b^{m}=$ $b^{m} a^{n}$ holds for all $a, b \in S$ and $m, n \geq 2$, while these identities hold in a commutative semigroup. 
It is well known that the $A G$-groupoids have many applications in the structure theory of semigroups. In particular, P.Holgate [3] called an $A G$-groupoid a simple invertive groupoid. We observe that a commutative group $(S, \circ)$ will become an $A G$-groupoid $(S, \cdot)$ under the following relation $a \cdot b=b \circ a^{-1}$, see( [7] and [8]). Clearly,an $A G$-groupoid $(S, \cdot)$ will become a semigroup under a new binary operation "o" defined on $S$. Thus, there exists an element $a \in S$ such that $x \circ y=(x a) y$ for all $x, y \in S$.

It is easy to show that the binary operation "o" is an associative operation and hence we have the following equality:

$$
\begin{aligned}
(x \circ y) \circ z & =(((x a) y) a) z=(z a)((x a) y)=(x a)((z a) y) \\
& =(x a)((y a) z)=x \circ(y \circ z) .
\end{aligned}
$$

Obviously, the system " $S, \circ)$ " forms a semigroup under the binary operation "o". In connection with the above discussion, the following example of a non-associative system is an interesting and useful example of an $A G$-groupoid.

In the following, we consruct an example of an $A G$-groupoid with a left identity.

Example 1.1. Let $S=\{1,2,3,4,5\}$ be a set with the binary operation "." defined on $S$ as follows:

\begin{tabular}{c|ccccc}
$\cdot$ & 1 & 2 & 3 & 4 & 5 \\
\hline 1 & 1 & 2 & 3 & 4 & 5 \\
2 & 5 & 1 & 2 & 3 & 4 \\
3 & 4 & 5 & 1 & 2 & 3 \\
4 & 3 & 4 & 5 & 1 & 2 \\
5 & 2 & 3 & 4 & 5 & 1
\end{tabular}

Then we can easily verify that the system " $(S, \cdot)$ " forms an $A G$-groupoid with a left identity 1.

We now call a subset $I$ of $S$ a left (right) ideal of $S$ if $S I \subseteq I$ and $(I S \subseteq I$ ), and a left (right) ideal of $S$ is said to be a minimal left (right) ideal of $S$ if $I$ does not contain any other left (right) ideal other than itself.

According to D. Rees [22], the kernel $K$ of $S$ may be described as the intersection of all two sided ideals of $S$. Later on, A. H. Clifford [2] noticed that if $M$ is a minimal ideal of a groupoid $S$ then $M$ is the kernel of $S$. Moreover, $K$ is a simple sub-groupoid of $S$. We describe here the non-associative structure of $S$. We shall call an $A G$-groupoid $S$ left (right) simple if $S$ does not contain any proper left (right) ideals of $S$. If $S$ is an $A G$-groupoid with left identity then $S a$ is a principal left ideal of $S$ generated by $a$ for all $a \in S$, and we can 
also show that $S a$ is an ideal of $S$ for all $a \in E(S)$, where $E(S)$ is the set of all the idempotents of $S$.

In the following example, we construct an $A G$-groupoid $S$ with a kernel $K$ of $S$.

Example 1.2. Let $S=\{1,2,3,4,5,6\}$ be a set on which a binary operation "." defined as follows:

\begin{tabular}{c|cccccc}
$\cdot$ & 1 & 2 & 3 & 4 & 5 & 6 \\
\hline 1 & 3 & 3 & 3 & 3 & 3 & 3 \\
2 & 3 & 3 & 3 & 3 & 3 & 3 \\
3 & 3 & 3 & 3 & 3 & 3 & 3 \\
4 & 3 & 3 & 3 & 3 & 3 & 3 \\
5 & 3 & 3 & 3 & 3 & 3 & 3 \\
6 & 3 & 2 & 3 & 3 & 3 & 3
\end{tabular}

It is celar to see that the above system $(S, \cdot)$ forms an $A G$-groupoid $S$. Obviously, $\{2,3\}$ is an ideal of $S$ and $\{3\}$ is a minimal ideal of $S$ and is also the kernel of $S$.

\section{Minimal Ideals and the Kernel of an $A G$-Groupoid}

The left and right minimal ideals of a semigroup and a ring have been extensively studied and investigated by L. M. Gluskin and O. Steinfeld in 1978, see [3]. We now begins with several lemmas of minimal ideals of an $A G$-groupoid $S$.

Lemma 2.1. Let $S$ be an $A G$-groupoid with left identity containing a minimal ideal $M$. Then $M$ is the kernel of $S$.

Proof. Suppose that $A$ is an ideal of $S$. Then $A M \subseteq A \cap M$ implies $A \cap M$ is non empty and so $A M \subseteq M$ and also $A M \subseteq A$ but since $M$ is minimal, $M=$ $A M \subseteq A$. This implies $M \subseteq A$ and $M$ is hence contained in every ideal of $S$, that is, $M$ is the kernel of $S$.

Lemma 2.2. Let $S$ be an $A G$-groupoid with a left identity. Then the kernel $K$ of $S$ is a simple $A G$-groupoid.

Proof. Let $K$ be the kernel of $S$ with left identity. If $A$ is an ideal of $K$, then $((K A) K)^{2}$ is obvious an ideal of $S$ contained in $K$, but since $K$ is the intersection of all the ideals of $S, K \subseteq((K A) K)^{2}$. This implies $((K A) K)^{2}=K$ 
and since $((K A) K)^{2} \subseteq A \subseteq K, A=K$. This proves that $K$ is a simple $A G$-groupoid.

We state below a crucial Lemma of a minimal left ideal of an $A G$-groupoid.

Lemma 2.3. Let $S$ be an $A G$-groupoid with left identity and $L$ a minimal left ideal of $S$. Then $L c$ is a minimal left ideal of $S$, for all $c \in S$.

Proof. Let $L$ be a minimal left ideal of $S$ with left identity. Then, it is trivial to see that $L c$ is a left ideal of $S$ for all $c \in S$, since $S(L c)=(S e)(L c)=$ $(S L)(e c) \subseteq L c$. Now, let $M$ be a minimal left ideal of $S$ contained in $L c$. If $L_{1} \subseteq L$ is the set of all elements $l_{1}$ of $S$ such that $l_{1} c \in M$ and $M=L_{1} c$, then we see immediately that $S\left(l_{1} c\right) \subseteq M$. This implies that $S\left(l_{1} c\right) \subseteq L_{1} c$ and we further deduce that $\left(S l_{1}\right)(e c) \subseteq L_{1} c$, for every $l_{1} \in L_{1}$, that is $\left(S L_{1}\right) c \subseteq L_{1} c$. This is clearly a contradiction. Thus, $L c$ is a minimal left ideal of $S$.

In corresponding to Lemma 2.3, we are able to give the following Lemma.

Lemma 2.4. Let $S$ be an $A G$-groupoid with left identity containing a zero element 0 . If $L$ is a minimal left ideal of $S$ and $c$ is an arbitray element of $S$ then either $L c$ is a minimal left ideal of $S$ or $L c=\{0\}$.

Proof. Let $L \neq\{0\}$ be a minimal left ideal of $S$. Then, $L c$ is clearly a minimal left ideal of $S$, for all $c \in S$. The proof of this Lemma is similar to Lemma 2.3. Hence $L c$ is a minimal left ideal of $S$ and $L c \neq\{0\}$.

\section{0-Minimal Ideals and the Class Sum of Left Minimal Ideals}

In this section, we call an ideal of an Abel-Grassmann groupoid $S$ 0-minimal if it is minimal in the set of all non-zero ideals of $S$. The set union of all minimal left ideals of $S$ is called the class sum of all minimal left ideals of $S$ and is denoted by $\Sigma$.

For o-minimal left ideal of an $A G$-groupoid $S$. We have the following Lemma.

Lemma 3.1. Any member in the set union $L S$ of all 0-minimal left ideals of $S$ is of the form $L x$ for some $x \in S$.

For 0-minimal left ideals of an $A G$-groupoid $S$, we futher have the following lemmas. 
Lemma 3.2. Let $L^{\prime}$ be any 0 -minimal left ideal of $S$ contained in $L S$. Then there exists some $x$ in $S$ such that $L^{\prime}=L x$.

Proof. Since every left ideal of $L S$ is of the form $L x$ for some $x \in S$ and all the 0-minimal left ideals of $S$ are 0 -disjoint. Let $L^{\prime}$ be some 0 -minimal left ideal of $S$ contained in $L S$. Then, there exists some $x$ in $S$ such that $L^{\prime}=L x$.

Lemma 3.3. If $x \in L S \backslash\{0\}$, then $S x$ is an 0-minimal left ideal of $S$ contained in $L S$ and $S x=L y$ for $y \in S$.

Proof. By Lemma 2.4, we deduce immediately that $L S$ is the set union of all 0-minimal left ideals of $S$ which are of the form $L y$ for any $y \in S$. Now let $x \in L S \backslash\{0\}$, then $L y$ is in $L S$ such that $x \in L y$ and the equation $l y=x$ has a solution in $L S$ for $y \in S$ and $l \in L \backslash\{0\}$. Thus $S(l y)=S x$ which implies $(S l) y=S x$, and since $S l$ is a left ideal of $S$ contained in $L$ and $L$ is an 0-minimal left ideal of $S$. Thus, $S l=L$. This implies $L y=S x$ for $y \in S$. Since $L y$ is an 0-minimal left ideal of $S, S x$ is 0-minimal left ideal of $S$.

The following lemmas are useful lemmas for minimal ideals of an $A G$ groupoid $S$ with left identity.

Lemma 3.4. Let $M$ be an 0-minimal ideal of $S$. Then either $M$ is an 0 -simple $A G$-subgroupoid or $M^{2}=\{0\}$.

Proof. The proof of the above lemma is exactly the same as in [18].

Lemma 3.5. Let $L$ be an 0-minimal left ideal of $S$ containing a zero element 0 and $L^{2} \neq\{0\}$. Then $L=S a$, for all $a \in L \backslash\{0\}$.

Proof. Suppose that $L$ is an 0 -minimal left ideal of $S$ and $L^{2} \neq\{0\}$. Now let $a$ be any non-zero element of $L$. Then $S a \subseteq L$ is a left ideal of $S$. Hence, either $S a=\{0\}$ or $L=S a$, for all $a \in L \backslash\{0\}$.Suppose that $S a=\{0\}$. Then, $a=0$. This is clearly impossible. Thus, $L=S a$ for all $a \in L \backslash\{0\}$.

For the o-minimal left ideals of the groupoid $S$, we deduce the following corollary.

Corollary 3.6. If $L$ is an 0 -minimal left ideal of $S$ containing an idempotent of $S$, then $L$ is an ideal of $S$ and $L=S a$, for all $a \in E(L)$.

Proof. Suppose that $a \neq 0$ belongs to $L$ and is also an idempotent. Then $S a$ is an ideal of $S$ and $S a \subseteq L$ but since $L$ is 0 -minimal left ideal of $S$, we see either $L=S a$ or $S a=\{0\}$ for all $a \in L \backslash\{0\}$. If $S a=\{0\}$ then $a=0$, contradicts to our hypothesis. Thus, $L=S a$. 
We now consider the minimal ideals of an $A G$-groupoid $S$.

We start with the following Lemma.

Lemma 3.7. Let $S$ be an $A G$-groupoid with left identity and $A$ a two sided ideal of $S$ containing every minimal left ideal of $S$. Then $L \subseteq A$ for any minimal left ideal $L$ of $S$.

Proof. Let $L$ be a minimal left ideal of $S$. If $A$ is a two sided ideal of $S$, then, $L A \subseteq A, A L \subseteq A$ and $A L \subseteq L$. Obviously, $A L$ is an left ideal of $S$ contained in $A$ and $L$. But since $L$ is a minimal left ideal of $S, A L=L$. Hence, we have shown that $L \subseteq A$.

For the class sum of all minimal left ideals of $S$, we have the following theorem.

Theorem 3.8. Let $S$ be an $A G$-groupoid with left identity. If $S$ contains at least one minimal left ideal and $\Sigma$ is the class sum of all minimal left ideals of $S$, then $\Sigma$ is the kernel of $S$.

Proof. Suppose that $\Sigma$ is the class sum of all minimal left ideals of $S$. Then, $\Sigma$ is an left ideal of $S$. Since by hypothesis, $\Sigma \neq \phi$ and so for every $c \in S, \Sigma c$ is also a left ideal of $S$, and for each $k \in \Sigma$ there exists some minimal left ideal $L$ of $S$ such that $k \in L$ and $k c \in L c \forall c \in S$, where $L c$ is a minimal left ideal of $S$. Hence, $k c \in \Sigma$ which implies $(k c) S \subseteq \Sigma$. This leads to $\Sigma S \subseteq \Sigma$. Thus, $\Sigma$ is a right ideal of $S$ and hence a two sided ideal of $S$. By the above Lemma, $\Sigma$ is contained in every ideal of $S$ and therefore $\Sigma$ itself is an ideal of $S$. We hence conclude that $\Sigma$ is contained in every ideal of $S$. Thus, $\Sigma$ is contained in the intersection of all ideals of $S$. In other words, $\Sigma$ is the kernel of $S$.

For the left ideals of an $A G$-groupoid $S$, we have the following Lemmas.

Lemma 3.9. Let $S$ be an $A G$-groupoid. Then every left ideal of the kernel $K$ of $S$ is still a left ideal of $S$.

Proof. Let $K$ be the kernel of $S$ and $A$ a left ideal of $K$, that is $K A \subseteq A$. Since each element $a$ of $A$ belongs to some minimal left ideal $L$ of $S, K a$ is a minimal left ideal of $S$ contained in $L$ for every $a \in A$ but since $L$ is the minimal left ideal of $S, K a=L$. This implies $a \in K a$ and hence, we further deduce that $A \subseteq K A$. Thus $A=K A$ as required.

Remark 3.10. Every minimal left ideal of $S$ is also a minimal left ideal of $K$ and vice versa. 


\section{Minimal Left Ideals}

In this section, we consider the minimal left ideals of an $A G$-groupoid $S$.

We first give the following Lemma.

Lemma 4.1. Every left ideal $A$ of an $A G$-groupoid $S$ contains at least one minimal left ideal of $S$.

Proof. Let $K$ be the class sum of all the minimal left ideals of $S$. If $A$ is an left ideal of $S$, then by Lemma $2.2, K A$ is also a left ideal of $S$ contained in $K$. But $K A=A$, we therefore conclude that $A \subseteq K$. This shows that $A$ contains at least one left ideal of $S$.

For the minimal left ideals of an $A G$-groupois $S$, We have the following theorem.

Theorem 4.2. A minimal left ideal $L$ of $S$ is a left simple $A G$-subgroupoid.

Proof. Suppose that $L$ is a minimal left ideal of $\mathrm{S}$ and $a \in L$. Then $L a$ is a minimal left ideal of $S$ contained in $L$. But since $L$ is minimal, $L a=L$. Thus $L$ is a left simple $A G$ sub-groupoid.

In the remaining part of this paper, we simply denote the minimal left ideal of $S$ by $L$. For the minimal right ideal $R$ of $S$, we have $R L=L$.

Lemma 4.3. If $a \in R$ and $b \in R L$, then the equation $a^{2} x=b$ has a solution $x$ in $R L$.

Proof. Suppose that $a \in R$. Then, $a^{2} \in R$. Now, it is obvious that $a^{2} R$ is a right ideal of $S$ contained in $R$. Since $R$ is a minimal right ideal of $S, a^{2} R=R$. This implies $\left(a^{2} R\right)(e L)=R L$ and we further deduce that $\left(a^{2} e\right)(R L)=R L$, that is, $a^{2}(R L)=R L$. Thus the equation $a^{2} x=b$ has a solution $x$ in $R L$ for all $b \in R L$.

Remark 4.4. If $S$ is an $A G$-groupoid with a left identity $e$ and $R$ is a right ideal of $S$, then $R e=e R=R$.

Lemma 4.5. If $a \in L$ and $b \in R L$ then the equation $x a=b$ has a solution $x$ in $R L$ for all $b \in R L$.

Proof. Suppose that $L$ is a minimal left ideal of $S$. Clearly, $L a$ is a minimal left ideal of $S$ and if $a \in L$ then $L a \subseteq L$. But since $L$ is minimal, $L a=L$ for 
all $a \in L$ which implies $R(L a)=R L$, and thereby, $(R L) a=R L$. Thus, $x a=b$ has a solution $x$ in $R L$ for every $b \in R L$.

Corollary 4.6. If $a \in L$ and $b \in R L$, then the equation $x a^{2}=b$ has a solution $x$ in $R L$, for all $b \in R L$.

The following Lemma is an easy consequence of the above Corollary.

Lemma 4.7. Let $S$ be a groupoid with left identity. If $a \in R \cap L$ and $b \in R L$. then the equations $a^{2} x=b$ and $y a^{2}=b$ has solutions $x, y$ in $R L$.

\section{Nil and Nipotent Ideals}

We state below the defintion of nil-ideals in an $A G$-groupoid.

Definition 5.1. Let $S$ be an $A G$-groupoid with left idetity. Then an ideal $A$ (left or right) of $S$ is said to be a nill-ideal of $S$ if for each $a \in A$, there exists some $n \in N$ such that $a^{n}=\underbrace{(\ldots(((a a) a) a) \ldots) a}_{n-\text { times }}=0$.

In the following theorem, we consider an $A G$-groupoid without nilpotent ideals.

Theorem 5.2. Suppose that an $A G$-groupoid $S$ does not contain any nilpotent ideals. Then every minimal ideal of $S$ is simple.

Proof. Let $M$ be a minimal ideal of $S$. Suppose that $B$, properly contained in $M$, is an ideal of $M$. Then, $M B M \subseteq M$ is a left ideal of $M$. In this case, $(M B M)^{2}$ is clerly an ideal of $S$ contained in $M$, but $M$ is minimal and therefore either $(M B M)^{2}=\{0\}$ or $(M B M)^{2}=M$. In either case, $(M B M)^{2}=\{0\}$ is not possible since $S$ does not contain any nilpotent ideals. Hence we deduce that $(M B M)^{2}=M$. This implies that $M \subseteq B$. Thus, $M=B$ and consequently, $M$ is simple.

In the following lemmas, we further study the left minimal ideals of an $A G$-groupoid $S$.

Lemma 5.3. If every ideal $A$ (left or right) of $S$ contains an idempotent then $S$ contains no non-trivial nil-ideals.

Proof. Suppose that $L$ is a left ideal of $S$ containing an idempotent $a$ of $S$. Now, we assume that $L$ is a nill-ideal of $S$, that is, for each element of $L$, there 
exists a positive integer $n$ such that $a^{n}=\underbrace{(\ldots(((a a) a) a) \ldots) a}_{n \text {-times }}=0$ but from our hypothesis, we know that $a^{2}=a$, for some $a \in A$, a contradiction. Thus, $S$ does not contain any nil-ideals.

Lemma 5.4. Let $M$ be a minimal ideal of $S$. Then every left ideal of $M$ is also a minimal left ideal of $S$.

Proof. Let $M$ be a minimal ideal of $S$. Then, by Lemma 3.7, $M$ contains at least one minimal left ideal $L$ of $S$. Now let $B \neq\{0\} \subseteq L$ be a left ideal of $M$, that is, $M B \subseteq B$ and $M B$ is an left ideal of $S$. Hence, $M B \subseteq L$, but $L$ is a minimal left ideal of $S$ and therefore, $L=M B \subseteq B$. This leads to $L \subseteq B$. Thus $L=B$.

The following results in $A G$-groupoids are similar to the well known classical results in semigroups.

Lemma 5.5. Let $S$ be an $A G$-groupoid $S$ with left identity. Then $S a$ is a principal ideal of $S$ generated by a for all $a \in E(S)$, where $E(S)$ is the set of all idempotents of $S$.

Proof. Suppose that $S$ has a left identity $e$, and $a$ an idempotentof $S$. Then, $(S a) S=\left(S a^{2} S\right)(S e)=(S S)\left(a^{2} e\right) \subseteq S a^{2} S=S a$. Hence, we can verify that $S a$ is a right ideal of $S$, and this proves that $S a$ is an ideal of $S$.

Lemma 5.6. Let $S$ be an $A G$-groupoid with left identity. Then $\left(S a^{2}\right) S=$ $S a^{2}$, for all $a \in S$.

Proof. Let $S$ be an $A G$-groupoid with a left identity $e$. Then

$$
S a^{2} S=\left(S a^{2}\right)(S e)=(S S)\left(a^{2} e\right)=S a^{2} .
$$

\section{Characterizations of 0-Simple and Simple $A G$-Groupoids}

In this section, we give a characterization theorem for 0 -simple and simple $A G$-groupoids.

Theorem 6.1. An AG-groupoid $S$ with left identity is 0 -simple if and only if $S a^{2}=S$ for all $a \in S \backslash\{0\}$.

Proof. By Lemma 5.6, we have $\left(S a^{2}\right) S=S a^{2}$ for all $a \in S$. The rest of the proof is exactly the same as in [18]. We hence omit the details. 
Corollary 6.2. An $A G$-groupoid $S$ with left identity is simple if and only if $S a^{2}=S$ for all $a \in S$.

Proof. The proof of the above corollary is exactly the same as in [18] and we hence omit the details.

The following lemmas are some useful lemmas for minimal ideals of an $A G$ groupoid $S$ with left identity.

Lemma 6.3. Let $M$ be an 0-minimal ideal of $S$. Then either $M$ is an 0 -simple $A G$-subgroupoid or $M^{2}=\{0\}$.

Proof. The proof of the above Lemma is exactly the same as in [18].

Lemma 6.4. Let $L$ be a 0 -minimal left ideal of $S$ containing a zero element 0 and $L^{2} \neq\{0\}$. Then $L=S a$, for all $a \in L \backslash\{0\}$.

Proof. Suppose that $L$ is an 0-minimal left ideal of $S$ and $L^{2} \neq\{0\}$. Now let $a$ be any non-zero element of $L$. Then $S a \subseteq L$ is a left ideal of $S$. Hence, either $S a=\{0\}$ or $L=S a$, for all $a \in L \backslash\{0\}$.Suppose that $S a=\{0\}$. Then, $a=0$. This is clearly impossible. Thus $L=S a$ for all $a \in L \backslash\{0\}$.

Corollary 6.5. If $L$ is an 0 -minimal left ideal of $S$ containing an idempotent of $S$. then $L$ is an ideal of $S$ and $L=S a$, for all $a \in E(L)$.

Proof. Suppose that $a \neq 0$ belongs to $L$ and is also an idempotent of $S$. Then $S a$ is clearly an ideal of $S$ and $S a \subseteq L$ but since $L$ is 0-minimal left ideal of $S$, we see that either $L=S a$ or $S a=\{0\}$ for all $a \in L \backslash\{0\}$. If $S a=\{0\}$ then $a=0$, which contradicts to our hypothesis. Thus $L=S a$.

The proof of the following corollary is trivial and we hence omit the details.

Corollary 6.6. Every left ideal of $S$ containing an idempotent contains an ideal of $S$.

For 0-minimal ideals, we have the following additional Lemmas.

Lemma 6.7. If $M$ is an 0 -minimal ideal of $S$ and $M^{2} \neq\{0\}$. Let $L \neq\{0\}$ contained in $M$ be a left ideal of $S$. Then either $L^{2}=M$ or $L^{2}=\{0\}$.

Proof. Suppose that $M$ is an 0 -minimal ideal of $S$ and $M^{2} \neq\{0\}$. We assume that $L \neq\{0\}$ is a left ideal of $S$ contained in $M$. Then, ${ }^{2} \subseteq M$ is an ideal of $S$. But since $M$ is minimal and so either $L^{2} \subseteq M$ or $L^{2}=\{0\}$. 
Lemma 6.8. Let $M$ be an 0-minimal ideal of $S$ containing at least one 0-minimal left ideal of $S$. Then $M$ is the set union of all the 0-minimal left ideals of $S$ contained in $M$.

Proof. Let $M$ be an 0-minimal ideal of $S$ and $M$ containing an 0-minimal left ideal $L$ of $S$. Now suppose that $A \subseteq M$ is the union of all 0-minimal left ideals of $S$ contained in $M$. Clearly, $A$ is a left ideal of $S$. Now let $a \in A \backslash\{0\}$ and $c \in S$. Then, by the definition of $A, a$ belongs to some 0-minimal left ideal $L$ of $S$, that is, $a \in L$, and $a c \in L c$. Since $L$ is an 0-minimal left ideal of $S$. Therefore, $L c$ is also an 0 -minimal left ideal of $S$. Thus, $L c \subseteq M$. This implies $a c \in M$ and $a c \in A$ as well which further implies that $A c \subseteq A$ for all $c \in S$. Hence, $A S \subseteq A$, that is, $A$ is the right ideal of $S$ and hence an ideal of $S$. As $A \subseteq M$ but $M$ is 0-minimal, and therefore $M=A$.

Let $A$ be a non empty subset of $S$. Then the intersection $K$ of all the left ideals $L$ of $S$ containing $A$ is a left ideal. Hence $A \subseteq K$ as well.

Lemma 6.9. Suppose that $S$ contains a left identity $e$ and $A$ is a nonempty subset of $S$. Then $A \cup S A$ is a left ideal of $S$ containing $A$.

Proof. Let $A$ be a non-empty subset of $S$. Then the following equalities hold:

$$
\begin{aligned}
S(A \cup S A) & =S A \cup S(S A)=(S A) \cup(S S)(S A)=(S A) \cup(A S)(S S) \\
& =(S A) \cup(A S) S=(S A) \cup(S S) A=S A \cup S A=S A \\
& \subseteq A \cup S A .
\end{aligned}
$$

From the above equality, $A \cup S A$ is a left ideal of $S$ containing $A$ and we call it the left ideal of $S$ generated by $A$. It is now clear that $A \cup(S A) \cup(A S) \cup(S A S)$ is also an ideal of $S$ generated by $A$. If $A$ contains only one element $a$, then we write $A=\langle a\rangle$, and $A \cup S A=S a$.

In summarizing the above results, we state a theorem of an $A G$-groupoid $S$ without nipotent ideals.

Theorem 6.10. Let $S$ be an $A G$-groupoid with left identity and contains no nilpotent ideals. Then every minimal left ideal of $S$ is contained in some minimal two sided ideals of $S$.

Proof. Let $L$ be minimal left ideal of $S$ and $L \neq\{0\}$. Then, $L \cup L S$ is an ideal of $S$ containing $L$. Since the following equality holds

$$
(L \cup L S) S=L S \cup(L S) S=(L S) \cup((e L) S) S=(L S) \cup((S L) e) S
$$




$$
\begin{aligned}
& \subseteq(L S) \cup(L e) S \subseteq(L S) \cup L \\
& \subseteq L \cup L S .
\end{aligned}
$$

Thus $L S \cup L$ is a right ideal of $S$, and hence an ideal of $S$. Now we still need to prove that $L \cup L S$ is a minimal ideal of $S$. Let $B \neq\{0\} \subseteq L \cup L S$ be an ideal of $S$. Then $B L \subseteq L$ is also a left ideal of $S$. But since $L$ is minimal, we either have $B L=\{0\}$ or $B L=L$. Suppose that $B L=\{0\}$. Then we deduce the following equation.

$$
B B \subseteq B(L \cup L S)=B L \cup B(L S)=\{0\} \cup(B e)(L S)=\{0\} \cup(B L) S=\{0\},
$$

Thereby $B^{2}=\{0\}$, which contradicts our hypotheisis. This leads to $B L=L$ and $B \subseteq L S$. Since $L c \subseteq B$, for all $c \in S$, and so $L S \subseteq B$, and consequently $L S=B$. This shows that $L \cup L S=B$ and $L \cup L S$ is a minimal ideal of $S$. In other words, every minimal left ideal of $S$ is contained in some minimal two sided ideal of $S$.This ends the proof.

Since quasi ideals of rings and semigroups have also been extensively studied by O.Stenfeld in his well known monograph [23].

In closing this paper, we ask for an $A G$-groupoid $S$ with left identity containing no nilpotent ideals, whether every minimal left quasi left ideal of $S$ will be properly contained in some miminal two sided quasi ideals of $S$ ?

\section{Acknowledgments}

The authors would like to thank the referee to her careful reading the draft of this paper.

\section{References}

[1] A.H. Clifford, Semigroup without nilpotent ideals, American Journal of Mathematics, 71, No. 4 (1949), 834-844.

[2] A.H. Clifford, Semigroups containing minimal ideals, American Journal of Mathematics, 70 (1948), 521-526.

[3] L.M. Gluskin, O. Steinfeld, Rings (semigroups) containing minimal (minimal) right and left ideals, Publ. Math. Debrecen, 25, No-s: 3-4 (1978), 275-280. 
[4] P. Holgate, Groupoids satisfying a simple invertive law, The Math. Stud., 61, No-s: 1-4 (1992), 101-106.

[5] J. Ježek, T. Kepka, A note on medial division groupoids, Proc. Amer. Math. Soc., 119, No. 2 (1993), 423-426.

[6] J. Ježek, T. Kepka, The equational theory of paramedial cancellation groupoids, Czech. Math. J., 50, No. 125 (2000), 25-34.

[7] M.A. Kazim, M. Naseeruddin, On almost semigroups, The Alig. Bull. Math., 2 (1972), 1-7.

[8] Q. Mushtaq, S. M. Yusuf, On LA-semigroups, The Alig. Bull. Math., 8 (1978), 65-70.

[9] Q. Mushtaq, S.M. Yusuf, On LA-semigroup defined by a commutative inverse semigroup, Math. Bech., 40 (1988), 59-62.

[10] Q. Mushtaq, Q. Iqbal, Decomposition of a locally associative LAsemigroup, Semigroup Forum, 41 (1990), 155-164.

[11] Q. Mushtaq, M. Khan, Ideals in AG-band and AG*-groupoid, Quasigroups and Related Systems, 14 (2006), 207-215.

[12] Q. Mushtaq, M. Khan, A note on an Abel Grassmann's-3-band, Quasigroups and Related Systems, 15 (2007), 295-301.

[13] Q. Mushtaq, M. Khan, M-systems in left almost semigroups, Southeast Asian Bull. Math., 33, No. 3 (2009), 321-327.

[14] Q. Mushtaq, M. Khan, Semilattice decomposition of locally associative $\mathrm{AG}^{* *}$-groupoids, Algebra. Colloq., 16, No. 1 (2009), 17-22.

[15] Q. Mushtaq, Madad Khan, Ideals in left almost semigroups, In: Proceedings of 4th International Pure Mathematics Conference (2003), 65-77.

[16] M. Khan, S. Anis, On semilattice decomposition of an Abel-Grassmann's groupoid, Acta Math. Sin. (Engl. Ser.), 28, No. 7 (2012), 1461-1468.

[17] M. Khan, S. Anis, K. P. Shum, Characterizations of left regular ordered Abel-Grassmann groupoids, Int. J. Algebra, 5, No-s: 9-12 (2011), 499-452.

[18] M. Khan, Some Studies in AG*-Groupoids, Ph.D. Thesis, Quaid-i-Azam University Islamabad, Pakistan (2008). 
[19] M. Khan, K. P. Shum and Shamas-ur-Rehman, On generalized fuzzy ideals and semigroups which are semilattices of left Groups, Southeast Asian Bull. Math., 37, No. 1 (2013), To Appear.

[20] P.V. Protic, N. Stevanovic, AG-test and some general properties of AbelGrassmann's groupoids, P.U.M.A., 6, No. 4 (1995), 371-383.

[21] N. Stevanović, P.V. Protić, Composition of Abel-Grassmann's 3-bands, Novi Sad J. Math., 34, No. 2 (2004), 175-182.

[22] D. Rees, On semigroups, Mathematical Proceedings of the Cambridge Philosphical Society, 36 (1940), 387-400.

[23] O. Steinfeld, Quasi-Ideals in Rings and Semigroups, With a foreword by L. Redei. Disquisitiones Mathematicae Hungaricae [Hungarian Mathematics Investigations], 10. Akademiai Kiado, Budapest (1978). xi+154. 
